\title{
Electronic Durability of Flexible Transparent Films from Type-Specific Single-Wall Carbon Nanotubes
}

\author{
John M. Harris, ${ }^{\S}$ Ganjigunte R. Swathi lyer, ${ }^{\S}$ Anna K. Bernhardt, ${ }^{\S}$ Ji Yeon Huh, ${ }^{\dagger}$ Steven D. Hudson, ${ }^{\dagger}$ \\ Jeffrey A. Fagan, ${ }^{\dagger}$ and Erik K. Hobbie ${ }^{*, \S}$ \\ ${ }^{\S}$ Department of Physics, Department of Coatings and Polymeric Materials, North Dakota State University, Fargo, North Dakota 58108, United States, and \\ ${ }^{\dagger}$ Polymers Division, National Institute of Standards and Technology, Gaithersburg Maryland 20899, United States
}

$\mathrm{R}$ esearch on the potential applications of single-wall carbon nanotubes (SWCNTs) has grown tremendously over the last two decades. Thin SWCNT films, in particular, show significant promise as transparent conductive coatings ${ }^{1}$ with outstanding electronic, mechanical, and optical properties. ${ }^{2-5} \mathrm{~A}$ particularly attractive feature of SWCNT films is that the physical properties can be tuned through the addition or subtraction of a relatively small number of nanotubes. This is because the percolation threshold for a collection of long slender rods scales inversely with aspect ratio, ${ }^{6}$ and hence can become exceptionally small for very high-aspect-ratio objects like SWCNTs. The electronic and optical properties of an individual SWCNT are dictated by its band structure, ${ }^{7}$ which is in turn determined by the chiral vector $(n, m)$ that characterizes the symmetry of rolling a 2D graphene sheet into a hollow tube. Unfortunately, as-produced nanotubes tend to contain a broad distribution of lengths, ranging from tens of nanometers up to thousands of micrometers, as well as a broad mixture of the two distinct species of electronic type, typically $1 / 3$ metallic and $2 / 3$ semiconducting. ${ }^{8}$ This polydispersity can have a profound influence on the physical properties of thin nanotube films. The critical role of nanotube aspect ratio, for example, in dictating the percolation behavior of thin SWCNT membranes has recently been demonstrated. ${ }^{6,9,10}$

Only very recently have improvements in SWCNT sorting and purification methods ${ }^{11-15}$ enabled the production of highly uniform SWCNT ensembles with specific length and/ or electronic type distributions. Thin films made from such materials hold tremendous potential for flexible electronics applications, including the replacement of indium tin oxide (ITO) in liquid crystal displays and

\section{ABSTRACT}
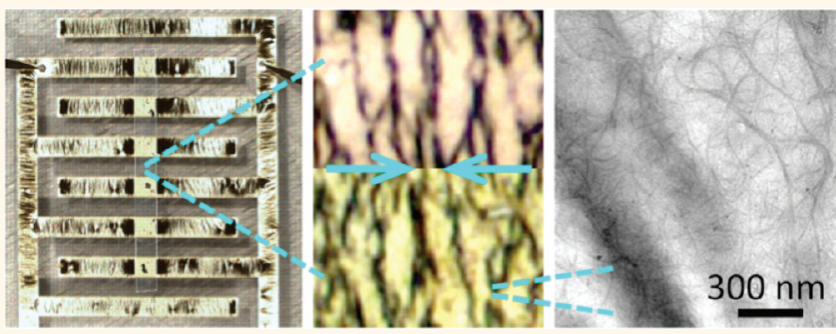

The coupling between mechanical flexibility and electronic performance is evaluated for thin films of metallic and semiconducting single-wall carbon nanotubes (SWCNTs) deposited on compliant supports. Percolated networks of type-purified SWCNTs are assembled as thin conducting coatings on elastic polymer substrates, and the sheet resistance is measured as a function of compression and cyclic strain through impedance spectroscopy. The wrinkling topography, microstructure and transparency of the films are independently characterized using optical microscopy, electron microscopy, and optical absorption spectroscopy. Thin films made from metallic SWCNTs show better durability as flexible transparent conductive coatings, which we attribute to a combination of superior mechanical performance and higher interfacial conductivity.

KEYWORDS: single-wall carbon nanotubes $\cdot$ metallic $\cdot$ semiconducting · flexible transparent electronics

photovoltaic devices. ${ }^{16}$ Metallic SWCNT membranes are among the most promising candidate materials in this regard, but the mechanical properties of these films are often only qualitatively described as "flexible", with a relative lack of any quantitative metrics. The wrinkling instability that emerges in compressed thin films on compliant substrates has emerged as a powerful tool for characterizing the mechanical properties of stiff membranes, and a variation on this theme has recently been used to study the bending mechanics ${ }^{17}$ of individual nanotubes. ${ }^{18}$ We recently used this approach to study the deformation mechanics of films assembled from length purified SWCNTs on *Address correspondence to
erik.hobbie@ndsu.edu.

Received for review November 11, 2011 and accepted December 10, 2011.

Published online December 11, 2011 $10.1021 / \mathrm{nn} 204383 \mathrm{t}$

C 2011 American Chemical Society 
soft polydimethylsiloxane (PDMS) substrates. ${ }^{9,10}$ By applying an empirical generalization of a continuum mechanical model for thin-film wrinkling ${ }^{19}$ to a wide range of compressed nanotube films, changes in membrane elasticity and sheet resistance were linked to strain-induced changes in microstructure and percolation threshold. These data suggest a remarkable degree of strain softening that couples intuitively to the electronic transport properties of the films., ${ }^{9,10}$ A number of important issues remain unresolved, however, regarding the microscopic origins of these effects, such as the fundamental roles of van der Waals interactions and electronic type in dictating the mechanical response of the films.

In this contribution, we consider the influence of compressive and cyclic strain on the electronic transport characteristics of type-purified nanotube membranes. Percolated networks of metallic and semiconducting SWCNTs are assembled as thin films on elastic polymer substrates, and the sheet resistance is measured as a function of applied strain through impedance spectroscopy. The topography, microstructure and optical transparency of the membranes are independently characterized using optical microscopy, electron microscopy, and optical absorption spectroscopy. We find significant differences in the electronic manifestations of thin-film wrinkling depending on the electronic type of the nanotubes, and we examine the underlying mechanisms. Our results are relevant to a number of ongoing efforts in transparent conducting films and flexible electronic devices.

\section{RESULTS AND DISCUSSION}

Our films are assembled from purified, type-sorted colloidal solutions of laser-ablation and electric arc discharge SWCNTs. The samples contain a broad distribution of lengths, as detailed in the Supporting Information. The solutions (Figure 1a) are deposited through vacuum filtration onto porous cellulose-ester filter paper supports and then transferred to elastic polymer substrates by back dissolving the filter paper with acetone. The electronic purity of the membranes is evident in the UV-vis-NIR absorption spectra of films deposited on quartz (Figure 1b), where the blue and brown-green curves represent metallic and semiconducting SWCNT films, respectively. There is a common graphitic absorption feature near $300 \mathrm{~nm}$ corresponding to the plasma frequency of the $\pi$ electrons. The metallic films show just two optical resonances, the $M_{22}$ just beyond the $\pi$-plasmon and the $M_{11}$ near $600-700 \mathrm{~nm}$. In contrast, the semiconducting films show three distinct resonances, $S_{11}, S_{22}$, and $S_{33}$. During the vacuum filtration process and subsequent rinsing to remove residual surfactant, individual SWCNTs assemble into bundles of 10 -to- $10^{3}$ nanotubes (Figure $1 \mathrm{c}$ ). Small-angle light scattering (SALS) from films deposited
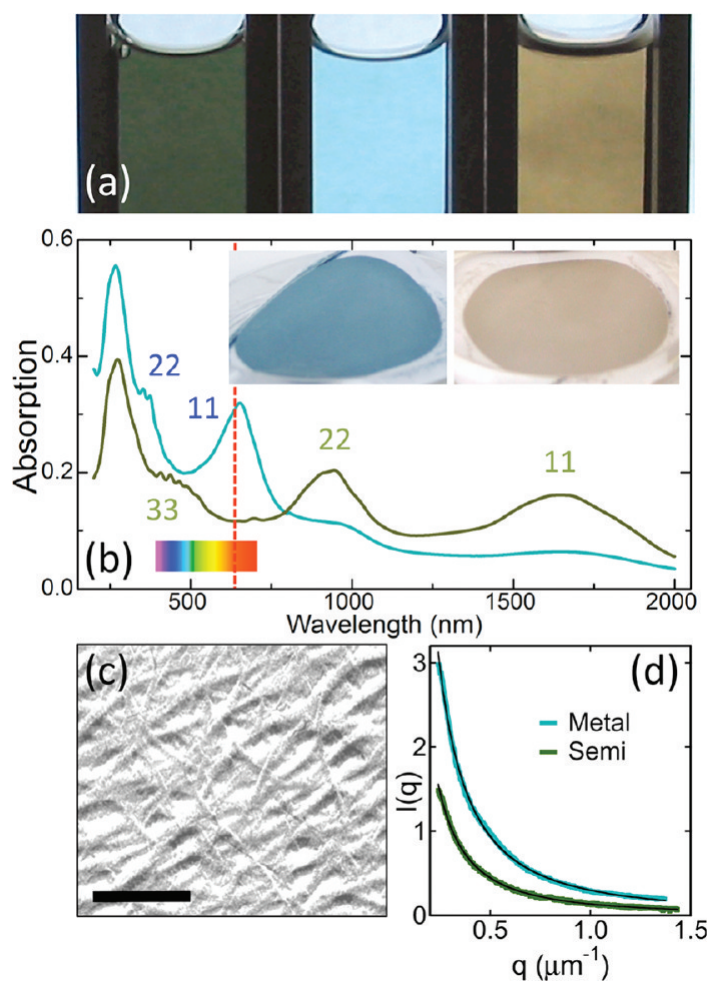

Figure 1. (a) Mixed-type colloidal SWCNT suspension (green) and the subsequent metallic (blue) and semiconducting (brown) fractions. (b) Optical absorption spectra of metallic (blue) and semiconducting (brown) films deposited on quartz with indicated resonant features. The visible spectrum and the laser line used to collect the scattering data in Figure 1d are indicated. The insets show typical typesorted membranes prepared on filter paper. (c) An unstrained semiconducting SWCNT film imaged with TEM (250 nm scale). (d) Small-angle light scattering profiles of type-sorted films on quartz with power-law fits as described in the text.

on quartz show that the quasi-2D bundle networks have a mass fractal structure (Figure 1d), with $I(q) \propto q^{-D}$ and a fractal dimension $D \approx 1.7$ independent of electronic type. The identical value of $D$ suggests that the morphology of the unperturbed networks is independent of electronic type.

The optical and electronic properties of the flexible films are characterized here as a function of film thickness. Three metallic films ( $h=20,28$, and $41 \mathrm{~nm}$ ) and one semiconducting film ( $h=35 \mathrm{~nm}$ ) were created with type-sorted aqueous colloidal solutions of electric arc SWCNTs commercially available from NanoIntegris. ${ }^{20}$ The remaining films were assembled from laser ablation SWCNTs type-sorted through an inhouse ultracentrifugation procedure as described elsewhere $^{21}$ and in the Supporting Information. Figure 2a shows the transmission spectra of the metallic films over the wavelength interval $530-730 \mathrm{~nm}$. Laser-ablation SWCNT films (denoted with shades of cyan) exhibit an $M_{11}$ interband feature at approximately $650 \mathrm{~nm}$, while the Nanolntegris films (displayed in blue) show a slight shift in $M_{11}$ to approximately $705 \mathrm{~nm}$ due to the 

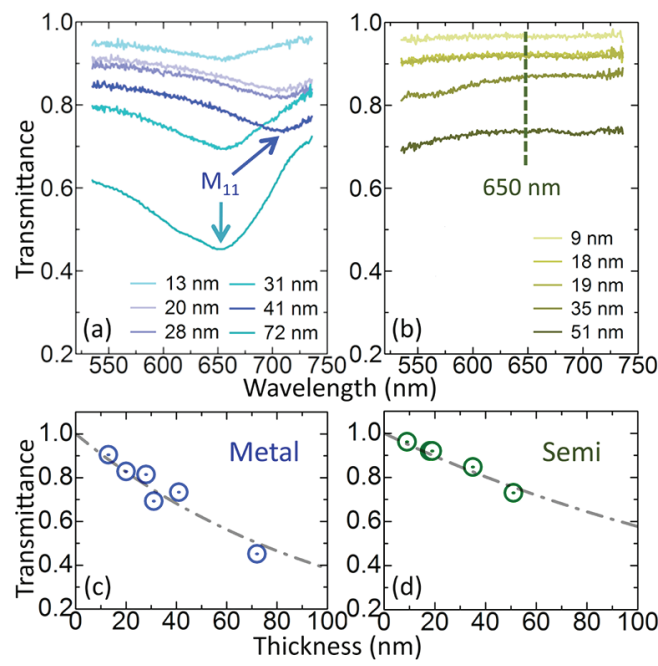

Figure 2. Optical transmittance spectra for (a) the metallic SWCNT films and (b) the semiconducting SWCNT films. (c) Transmittance as a function of film thickness, $h$, for the metallic SWCNTs at the $M_{11}$ resonance and (d) the semiconducting SWCNTs at $650 \mathrm{~nm}$. The curves are fits to an exponential decay.

slightly larger average diameter of the electric arc nanotubes. Transmission spectra of the semiconducting films are relatively featureless over the relevant wavelength interval. The transmittance in Figure $2 \mathrm{a}$ is plotted as a function of film thickness, $h$, and fit to an exponential decay, ${ }^{22,23}$

$$
T(h)=\mathrm{e}^{-A h}
$$

The extinction coefficients are $A=0.0096 \pm 0.001 \mathrm{~nm}^{-1}$ for the resonant $M_{11}$ metallic data (Figure $2 \mathrm{c}$ ) and $A=$ $0.0055 \pm 0.0007 \mathrm{~nm}^{-1}$ for the semiconducting data (650 nm, Figure 2d).

Gold electrodes were deposited across the surface of each film (Figure $4 \mathrm{f}$ ) and impedance spectroscopy was used to measure the complex sheet resistance as a function of strain on flexible PDMS substrates. The zerostrain sheet resistance is broken down into real and imaginary parts as a function of frequency for metallic and semiconducting films in Figure 3. The response is typical of percolated conducting networks, ${ }^{6}$ with a real part (solid) that is independent of frequency and an imaginary part (dashed) that increases linearly with frequency. The zero-strain sheet conductivity as a function of film thickness is fit to the expression ${ }^{24}$

$$
\sigma(h)=\sigma_{0}\left(h / h_{\mathrm{c}}-1\right)^{\alpha}
$$

where $\sigma_{0}$ is the critical amplitude, $h_{\mathrm{c}}$ is the film thickness at the percolation threshold, and $\alpha$ is the critical exponent. The metallic data (Figure $3 \mathrm{~b}$ ) yield $\sigma_{0}=3.3 \times$ $10^{-4} \square \Omega^{-1}$ and $h_{\mathrm{c}}=12 \pm 2 \mathrm{~nm}$ with a critical exponent of $0.92 \pm 0.1$, while the fit of the semiconducting data (Figure 3e) yields $\sigma_{0}=7.7 \times 10^{-5} \square \Omega^{-1}$ and $h_{\mathrm{c}}=6 \pm$ $2 \mathrm{~nm}$ with a critical exponent of $1.15 \pm 0.1$. Results from both film types are in agreement with accepted values
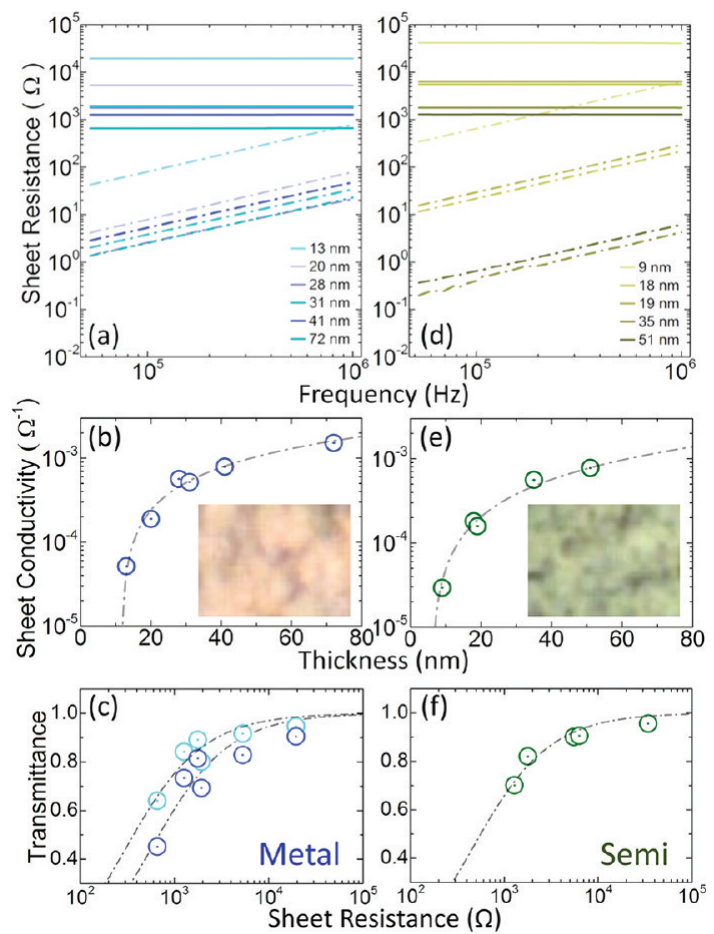

Figure 3. (a) Complex sheet resistance as a function of frequency, (b) sheet conductivity as a function of thickness, and (c) transmittance as a function of sheet resistance for the metallic films. $(d-f)$ Analogous plots for the semiconducting films. The real and imaginary parts are shown as solid and dashed curves, respectively, in panels a and d. Fits to eq 2 in panels $b$ and $e$ are shown as dashed curves. The fits in panels $c$ and $f$ are based on the standard model of conducting films described in the text, where cyan markers in Figure 3c represent off-resonant extinction. Reflection optical micrographs of the two film types are shown in Figure 3b,e.

of the critical exponent $(\alpha \approx 1)$ for percolated 2D conducting networks. ${ }^{25-29}$ Film thicknesses range from 9 to $72 \mathrm{~nm}$, much less than the wavelength of light, and the transmittance as a function of sheet resistance, $R_{\mathrm{s}}$ is fit to the expression 22,23

$$
T\left(R_{\mathrm{S}}\right)=\left(1+\frac{1}{2 R_{\mathrm{S}}} \sqrt{\frac{\mu_{0}}{\varepsilon_{0} \sigma_{\mathrm{op}}}}\right)^{-2}
$$

where $\varepsilon_{0}$ and $\mu_{0}$ are the permittivity and permeability of free space, respectively, $\sigma_{\mathrm{op}}$ is the optical conductivity, and $\sigma_{\mathrm{dc}}$ is the direct current conductivity. The ratio $\sigma_{\mathrm{op}} / \sigma_{\mathrm{dc}}$ quantifies the combined optical and electronic quality of the films. Our measurements yield a ratio of $1.52 \pm 0.3$ for metallic films (Figure 3c) and $1.24 \pm 0.3$ for semiconducting films (Figure 3e). An offresonant $(500 \mathrm{~nm})$ transparency measure for the metallic films gives a slightly better ratio $(0.8 \pm 0.3)$. All impedance spectroscopy measurements reported here were performed on compliant PDMS ( $E=1.8 \mathrm{MPa}$ and $v=0.48$, where $E$ and $v$ are the Young modulus and Poisson ratio, respectively). A 2-fold reduction in sheet conductivity is observed for films deposited on PDMS as opposed to quartz, which we attribute to SWCNT 

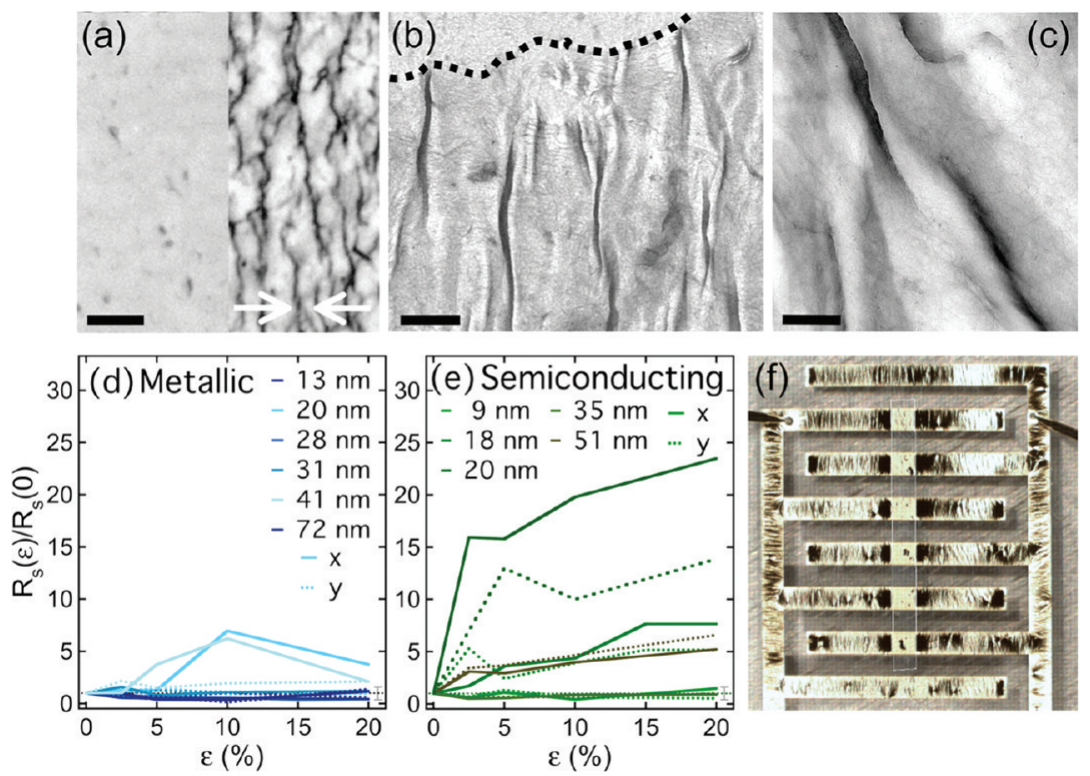

Figure 4. (a) Reflection optical micrograph of an unstrained $35 \mathrm{~nm}$ thick semiconducting film on quartz (left) and the same film on PDMS subjected to a $10 \%$ compressive strain (10 $\mu \mathrm{m}$ scale). (b) TEM image of the same film at $10 \%$ strain ( $2 \mu \mathrm{m}$ scale). The dotted curve delineates the edge of the film. (c) The same film viewed with TEM at higher magnification ( $300 \mathrm{~nm}$ scale). (d) Strain response of the sheet resistance for metallic films. (e) Strain response of the sheet resistance for semiconducting films. (f) Typical film configuration showing the electrode pattern.

network damage that occurs as a result of the swelling/ deswelling processes in the PDMS. ${ }^{9}$ When this factor is applied to the data in Figure 3, we find reasonable agreement with comparable measurements reported by Green et $a .^{23}$ For the relevant range of film thicknesses, reflection optical micrographs on transparent substrates appear pink for the metallic films and green for the semiconducting films due to constructive interference effects (insets, Figure 3b,e).

Our main interest here is the influence of strain on flexible electronic performance, which is uninvestigated for type-purified SWCNT films. Optical reflection micrographs in Figure 4a show an unstrained SWCNT film (left) and the same film under compression (right). Figure $4 \mathrm{~b}$ shows this same film imaged with transmission electron microscopy, where the dotted line delineates the edge of the membrane. A higher magnification TEM image of the same film is shown in Figure 4c. Variations in local film thickness are correlated with density variations across wrinkled regions of the compressed film. The underlying mechanics of a similar process are described in detail in two earlier contributions. ${ }^{9,10}$ Smaller bundles coarsen into larger bundles aligned normal to the direction of the applied strain, which gives the film a surface profile of dark regions surrounded by lighter webs of interconnected bundles. The wrinkling pattern in Figure 4a closely resembles the behavior we previously observed for length-purified mixed-type films ${ }^{9,10}$ and is strikingly similar to the pattern predicted for the elasto-plastic deformation of compressed stiff films on soft substrates. ${ }^{30}$
An interesting question is how these structural changes affect sheet conductivity. To address this, a large number of films were deposited on prestrained PDMS substrates and subjected to compression through the release of the strain. The density profile of a compressed film measured in TEM (Figure 4b,c) suggests that the SWCNTs align normal to the direction of prestrain. ${ }^{9,10}$ Two pieces of the same film were thus deposited on each PDMS substrate; one oriented parallel to the compression axis $(x)$ and the other oriented perpendicular $(y)$. Gold electrodes were then deposited on the surface of the film, as shown in Figure $4 \mathrm{f}$. The anisotropic sheet resistance was measured for films prepared at $0,2.5,5,10,15$, and $20 \%$ compressive strain in the $x-y$ (solid/dashed) plane for both metallic (Figure 4d) and semiconducting (Figure 4e) films. Statistically, for an ensemble of over 100 SWCNT films prepared on over 50 different PDMS substrates, the conductivity of the metallic films is more robust in response to compression; $12 \%$ of strained metallic films show $>2 \times$ anisotropic loss of conductivity, compared with $55 \%$ of strained semiconducting films. Both types show the strongest anisotropy at thicknesses just inside the percolation region, where the networks will be less developed and weaker.

These observations raise the question of durability under cyclic strain, which we investigated for a $30 \mathrm{~nm}$ metallic film and a $50 \mathrm{~nm}$ semiconducting film (Figure 5). Both samples were prepared at $10 \%$ compressive strain and then subjected to the strain sequence $7.5,5,0,7.5$, $5,0,7.5,5,0,10 \%$ by repeatedly stretching and relaxing the samples in a strain gauge (the $0 \%$ strain point in 


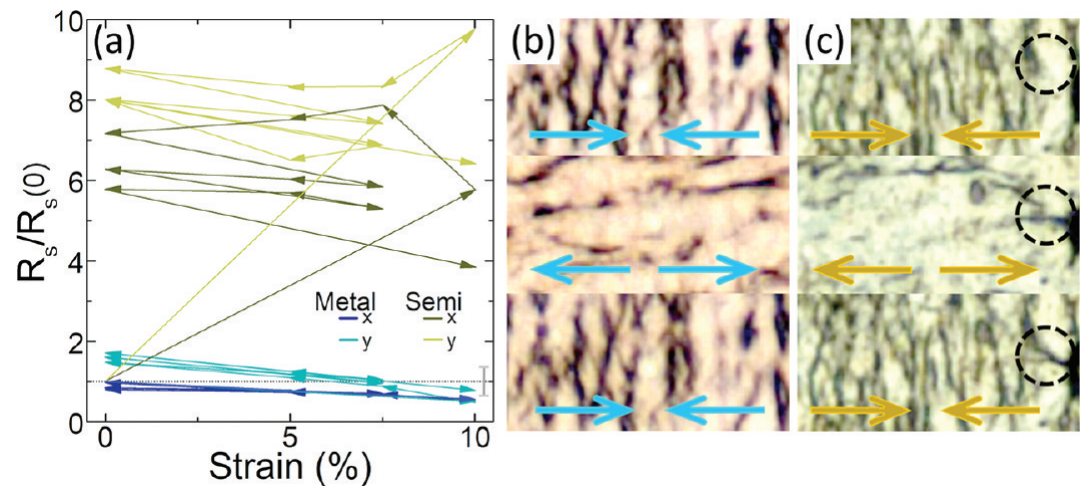

Figure 5. (a) Cyclic strain history of sheet resistance for the metallic and semiconducting films, where the data are normalized by the zero-strain values in Figure 3. (b) Reflection optical micrographs of the wrinkling topography under cyclic strain for a $30 \mathrm{~nm}$ thick metallic film, and (c) analogous images for a $50 \mathrm{~nm}$ thick semiconducting film. The strain sequence in the images is 10,0 , and $10 \%$, from top to bottom, where the semiconducting films show topographical change as indicated.

Figure 5 corresponds to the independent measurement in Figure 3). The resulting changes in conductivity are illustrated in Figure $5 \mathrm{a}$ and support the conclusion that cyclic strain has a larger impact on the semiconducting films. From top to bottom, the optical images in Figure 5 are taken at 10, 0, and 10\% strain, corresponding to cycle points 2,8 , and 12 . Comparing the top and bottom image in Figure $5 \mathrm{~b}$, the wrinkling pattern is nearly unchanged. The semiconducting film in Figure 5c exhibits a similar "memory", but new topography that emerges later in the cycle suggests continued structural change in the film. Insight into the underlying mechanics follows from a simple scaling analysis. In a linear elastic film, and to some approximation in a yielding SWCNT film, the characteristic wrinkling length scale is ${ }^{9,10} \lambda \propto h E_{f}^{1 / 3}$, where $E_{f}$ is the Young modulus of the film. Knowing the thickness ratio, and computing the average wrinkling length scale from optical micrographs, at $10 \%$ strain the modulus of the semiconducting film is a factor of 0.2 smaller than that of the metallic film, even though the semiconducting film is a factor of 5 deeper into the (unstrained) percolation regime. The loss of conductivity in the semiconducting film (Figure 5a) would thus appear to be accompanied by a significant loss in mechanical rigidity.

\section{CONCLUSIONS}

SWCNT-polymer composites are of potential interest for a broad range of emerging applications. ${ }^{31-34}$ Through impedance spectroscopy performed on flexible SWCNT membranes deposited on elastic polymer substrates, we have characterized the coupling between mechanical flexibility and electronic performance in thin metallic and semiconducting SWCNT films. Optical transmittance as a function of sheet resistance is consistent with high-quality films, which are well characterized by the concept of percolation in quasi2D thin fibrous networks. The zero-strain percolation threshold of the semiconducting films is approximately half that of the metallic films, while the semiconducting films are more fragile, suggesting a possible difference in the nature of van der Waals contact forces in the respective networks. Such forces play a critical role in the mechanical response of single-wall carbon nanotube networks. ${ }^{35-37}$ Although the length distribution of the semiconducting SWCNTs is slightly broader than that of the metallic SWCNTs (Supporting Information), a broader width would be expected to increase the percolation threshold, ${ }^{6}$ and the peak in the length distribution is relatively insensitive to electronic type. The results we present here thus suggest that the metallic films make better flexible transparent conductive coatings; they have higher conductivity/transmission and are more durable.

Both films remember the initial wrinkling configuration in subsequent cycles. Once a compressive strain is applied, and the film responds by folding, the same pattern persists through all future relaxation/compression cycles (Figure 5). In the case of the semiconducting SWCNTs, this pattern becomes augmented by additional topography that is indicative of further plastic change in the films. Since the microstructure of the films is known to evolve as the film deforms, ${ }^{9,10}$ the reproducibility of the pattern that emerges in the first strain experiment is a combined consequence of the initial quenched thickness fluctuations in the film and any subsequent strain-induced changes in microstructure. Although there are plastic changes in the metallic films, our data suggest that these occur primarily in the first compression. Additionally, since conductivity in the film only requires intimate electrical contact across the network and not rigidity, physical contact of plastically altered microstructural regions will be sufficient to maintain electrical percolation, as long as the interfacial conductivity is high. The interfacial conductance of type-specific SWCNT contacts has been measured ${ }^{38}$ and metallic-metallic contacts do indeed show better conductivity. Evidence for this can also be seen in Figure 4d, where there is a 
compressive restoration of the conductivity above $10 \%$ strain for the metallic films. On the basis of all of these observations, we suggest that metallic SWCNT films are more durable as flexible transparent conductors because (i) the metallic films themselves are more mechanically robust and (ii) the impedance between contacted metallic SWCNT bundles is smaller.

\section{EXPERIMENTAL METHODS}

Sample Preparation. Polymer substrates of polydimethylsiloxane (PDMS) were prepared at a 10:1 monomer/cross-linker ratio. The two components were mixed, poured into a mold, and placed in a vacuum oven for $1 \mathrm{~h}$ to remove air bubbles. Without breaking vacuum, the mold was then baked at $80^{\circ} \mathrm{C}$ for 2 hours. Substrates with dimensions $75 \mathrm{~mm} \times 25 \mathrm{~mm} \times 1.5 \mathrm{~mm}$ were cut from the mold and cleaned with ethanol. The two ends of a substrate were then clamped in a strain stage and a controlled stretch was applied up to a specific strain. Two SWCNT pieces of approximately $2 \mathrm{~mm} \times 10 \mathrm{~mm}$ were cut from the filter paper and placed SWCNT-face down onto the prestrained substrate, one oriented with the long edge normal to the direction of strain and the other oriented parallel. The sample was then subjected to a gentle acetone wash over the course of $1 \mathrm{~h}$ to remove the filter paper. This was followed by a $20 \mathrm{~min}$ ethanol rinse to remove any residual surfactant, and the test specimen was then placed in a vacuum for several hours to expedite drying. Once dry, the strain on the substrate was carefully released at a controlled and consistent rate, compressing the two films. The sample was then positioned on a mask and placed in a deposition chamber where $10 \mathrm{~nm}$ of chromium followed by $100 \mathrm{~nm}$ of gold was sputtered onto the sample without breaking vacuum (Figure 4f). Pieces of the SWCNT films were also deposited in a similar manner on quartz to measure spectra, scattering, and thickness.

Characterization. UV-vis-NIR spectroscopy was performed on a commercial spectrometer, and optical transparency analysis was performed on an optical microscope equipped with a spectrometer. Multiple spectra were captured at various locations across the surface of each film. An atomic force microscope (AFM) configured in tapping mode was used to collect several images at various points along the edges of each film, and the thickness was determined by analyzing the quartzSWCNT step height normal to the edge. Impedance spectroscopy, configured for a 2-probe capacitive frequency (CF) test and calibrated against a $1.01 \mu \mathrm{F}$ capacitor, was used to measure the complex impedance spectra for each sample. The CF measurement uses a $250 \mathrm{mV}$ amplitude over the frequency interval $1-1000 \mathrm{kHz}$. A reflection optical microscope was used to collect images of all tested gaps, and these images were used to compute geometrical factors to convert impedance to sheet resistance. A 4-point probe was then used to measure the zerofrequency sheet resistance of each sample, calibrated against an ITO standard. This 4-point measurement was used for the final calibration of each impedance trace from the low frequency plateau in the real part of the response. For cyclic strain experiments, optical measurements of the in-plane geometry as a function of strain were used to compute strain-dependent geometric factors. Measurements of the conductivity of the gold electrodes under cyclic strain showed negligible effects associated with strain-induced changes in electrode structure, contact, and topography. Static small-angle light scattering measurements were performed with a $30 \mathrm{~mW} \mathrm{He}-\mathrm{Ne}$ laser directed through unstrained films deposited on thin quartz. The scattered light was imaged on a screen equipped with a beam stop using a thermoelectrically cooled CCD, and measured background scattering from the quartz substrate was subtracted from the total intensity. The signal was then circularly averaged and reduced by the thickness of the film. Increased scattering in the metallic film (Figure 1d) is a consequence of the shape of the real part of the dielectric response and the nearresonant $\left(M_{11}\right)$ laser line. ${ }^{39}$ Unless otherwise indicated, error bars are the size of the data markers and represent the maximum instance of two standard deviations in uncertainty.
To image the membranes with transmission electron microscopy (TEM), SWCNT films on quartz or PDMS were first coated with evaporated $\mathrm{Pt} / \mathrm{C}$ at a shadowing angle of approximately $26^{\circ}$ (the height of an object is thus half of the length of its shadow). A thin evaporated coating of carbon was then applied at normal incidence. To detach the decorated nanotube membranes from the supporting substrates, a drop of aqueous poly(acrylic acid) (PAA) solution was placed on the film, allowed to dry overnight at $55^{\circ} \mathrm{C}$, and then removed the following day. Slow removal of the PAA typically resulted in a clean detachment of the membrane from the substrate. The detached sample was then placed PAA side down onto a water bath, dissolving the PAA and floating the SWCNT membrane on the water surface. After gentle washing, the film was retrieved on a copper 600 mesh grid. TEM was performed using a Philips EM400T operated at $120 \mathrm{kV}$ in bright-field mode with an objective aperture to enhance contrast. Images were recorded with a Cantega $2 \mathrm{k}$ CCD camera. In this mode, the darkness of a point in the image is correlated to the total mass (integrated thickness) at that location.

Acknowledgment. We thank Jan Obrzut, Chris Stafford, Jun Chung, Benjamin Forsythe, Steven Ruckdashel, Matthew Mumm, and Abby Mattson for assistance. E.K.H. acknowledges the support of the NSF through CMMI-0969155 (J.M.H.) and the DOE through DE-FG36-08G088160 (G.R.S.I.).

Supporting Information Available: Details of the SWCNT separation scheme and the measured SWCNT length distributions, with AFM images. This material is available free of charge via the Internet at http://pubs.acs.org.

\section{REFERENCES AND NOTES}

1. Blackburn, J. L.; Barnes, T. M.; Beard, M. C.; Kim, Y.-H.; Tenent, R. C.; McDonald, T. J.; To, B.; Coutts, T. J.; Heben, M. J. Transparent Conductive Single-Walled Carbon Nanotube Networks with Precisely Tunable Ratios of Semiconducting and Metallic Nanotubes. ACS Nano 2008, 2, 1266-1274.

2. Cao, Q.; Rogers, J. A. Ultrathin Films of Single-Walled Carbon Nanotubes for Electronics and Sensors: A Review of Fundamental and Applied Aspects. Adv. Mater. 2008, 21, 29-53.

3. Hu, L.; Hecht, D. S.; Grüner, G. Carbon Nanotube Thin Films: Fabrication, Properties, and Applications. Chem. Rev. 2010, 110, 5790-5844.

4. Kis, A; Zettl, A. Nanomechanics of Carbon Nanotubes. Phil. Trans. R. Soc. A 2008, 366, 1591-1611.

5. Zhang, D.-B.; Dumitrică, T. Elasticity of Ideal Single-Walled Carbon Nanotubes via Symmetry-Adapted Tight-Binding Objective Modeling. Appl. Phys. Lett. 2008, 93, 031919.

6. Simien, D.; Fagan, J. A.; Luo, W.; Douglas, J. F.; Migler, K.; Obrzut, J. Influence of Nanotube Length on the Optical and Conductivity Properties of Thin Single-Wall Carbon Nanotube Networks. ACS Nano 2008, 2, 1879-1884.

7. Saito, R.; Dresselhaus, G.; Dresselhaus, M. S. Physical Properties of Carbon Nanotubes; Imperial College Press: London, 1999.

8. Jorio, A. P.; Ribeiro, H. B.; Fantini, C.; Souza, M.; Vieira, J. P. M.; Furtado, C. A.; Jiang, J.; Saito, R.; Balzano, L.; Resasco, D. E.; et al. Quantifying Carbon-Nanotube Species with Resonance Raman Scattering. Phys. Rev. B 2008, 72, 075207.

9. Harris, J. M.; Iyer, G. R. S.; Simien, D. O.; Fagan, J. A.; Huh, J. Y.; Chung, J. Y.; Hudson, S. D.; Obrzut, J.; Douglas, J. F.; Stafford, C. M.; et al. Structural Stability of Transparent Conducting 
Films Assembled from Length Purified Single-Wall Carbon Nanotubes. J. Phys. Chem C 2011, 115, 3973-3981.

10. Hobbie, E. K.; Simien, D. O.; Fagan, J. A.; Huh, J. Y.; Chung, J. Y.; Hudson, S. D.; Obrzut, J; Douglas, J. F.; Stafford, C. M. Wrinkling and Strain Softening in Single-Wall Carbon Nanotube Membranes. Phys. Rev. Lett. 2010, 104, 125505.

11. Yanagi, K.; Miyata, Y.; Kataura, H. Optical and Conductive Characteristics of Metallic Single-Wall Carbon Nanotubes with Three Basic Colors; Cyan, Magenta, and Yellow. Appl. Phys. Express 2008, 1, 034003.

12. Fagan, J. A.; Becker, M. L.; Chun, J.; Hobbie, E. K. Length Fractionation of Carbon Nanotubes Using Centrifugation. Adv. Mater. 2008, 20, 1609-1613.

13. Arnold, M. S.; Suntivich, J.; Stupp, S. I.; Hersam, M. C. Hydrodynamic Characterization of Surfactant Encapsulated Carbon Nanotubes Using an Analytical Ultracentrifuge. ACS Nano 2008, 2, 2291-2300.

14. Tu, X.; Manohar, S.; Jagota, A.; Zheng, M. DNA Sequence Motifs for Structure-Specific Recognition and Separation of Carbon Nanotubes. Nature 2009, 460, 250-253.

15. Fagan, J. A.; Becker, M. L.; Chun, J.; Nie, P.; Bauer, B. J.; Simpson, J. R.; Hight-Walker, A.; Hobbie, E. K. Centrifugal Length Separation of Carbon Nanotubes. Langmuir 2008, $24,13880-13889$

16. Hu, L.; Hecht, D. S.; Grüner, G. Infrared Transparent Carbon Nanotube Thin Films. Appl. Phys. Lett. 2009, 94, 081103.

17. Nikiforov, I.; Zhang, D.-B.; James, R. D.; Dumitrică, T. Wavelike Rippling in Multiwalled Carbon Nanotubes under Pure Bending. Appl. Phys. Lett. 2010, 96, 123107.

18. Khang, D.-Y.; Xiao, J.; Kocabas, C.; MacLaren, S.; Banks, T.; Jiang, H.; Huang, Y. Y.; Rogers, J. A. Molecular Scale Buckling Mechanics in Individual Aligned Single-Wall Carbon Nanotubes on Elastomeric Substrates. Nano Lett. 2008, 8, 124-130.

19. Jiang, H. Q.; Khang, D.; Song, J.; Sun, Y.; Huang, Y.; Rogers, J. A. Finite Deformation Mechanics in Buckled Thin Films on Compliant Supports. Proc. Natl. Acad. Sci. 2007, 104, 15607-15612.

20. Certain equipment, instruments, or materials are identified in this paper in order to adequately specify the experimental details. Such identification does not imply recommendation by the National Institute of Standards and Technology nor does it imply the materials are necessarily the best available for the purpose.

21. Hobbie, E. K.; Fagan, J. A.; Obrzut, J.; Hudson, S. D. Microscale Polymer-Nanotube Composites. ACS Appl. Mater. Interfaces 2009, 1, 1561-1566.

22. Zhou, Y. X.; Hu, L. B.; Gruner, G. A Method of Printing Carbon Nanotube Thin Films. Appl. Phys. Lett. 2006, 88, 123109.

23. Green, A. A.; Hersam, M. C. Colored Semitransparent Conductive Coatings Consisting of Monodisperse Metallic Single-Walled Carbon Nanotubes. Nano Lett. 2008, 8, 1417-1422.

24. Hu, L.; Hecht, D. S.; Gruner, G. Percolation in Transparent and Conducting Carbon Nanotube Networks. Nano Lett. 2004, 4, 2513-2517.

25. Clerc, J. P.; Giraud, G.; Laugier, J. M.; Luck, J. M. The Electrical Conductivity of Binary Disordered Systems, Percolation Clusters, Fractals and Related Models. Adv. Phys. 1990, 39, 191-309.

26. Frank, D. J.; Lobb, C. J. Highly Efficient Algorithm for Percolative Transport Studies in Two Dimensions. Phys. Rev. B 1988, 37, 302-307.

27. Laugier, J. M.; Clerc, J. P.; Giraud, G.; Luck, J. M. AC Properties of 2D Percolation Networks: A Transfer Matrix Approach. J. Phys. A: Math. Gen. 1986, 19, 3153-3164.

28. Lajkó, P.; Turban, L. Percolation and Conduction in Restricted Geometries. J. Phys. A: Math. Gen. 2000, 33, 1683-1692.

29. Kholodenko, A. L.; Freed, K. F. Diffusion in Random Media as a Problem of Interacting Bose and Fermi Fields. J. Phys. A: Math. Gen. 1984, 17, L55-L59.

30. Yin, J.; Chen, X. Buckling Patterns of Thin Films on Compliant Substrates: The Effect of Plasticity. J. Phys. D: Appl. Phys. 2011, 44, 045401.
31. Kim, D.; Kim, Y.; Choi, K.; Grunlan, J. C.; Yu, C. Improved Thermoelectric Behavior of Nanotube-Filled Polymer Composites with Poly(3,4-ethylenedioxythiophene) Poly(styrenesulfonate). ACS Nano 2010, 4, 513-523.

32. Hermant, M.-C.; van der Schoot, P.; Klumperman, B.; Koning, C. E. Probing the Cooperative Nature of the Conductive Components in Polystyrene/Poly(3,4 ethylenedioxythiophene):Poly(styrene sulfonate)-Single-Walled Carbon Nanotube Composites. ACS Nano 2010, 4, 2242-2248.

33. De, S.; Lyons, P. E.; Sorrel, S.; Doherty, E. M.; King, P. J.; Blau, W. J.; Nirmalraj, P. N.; Boland, J. J.; Scardaci, V.; Joimel, J.; et al. Transparent, Flexible, and Highly Conductive Thin Films Based on Polymer-Nanotube Composites. ACS Nano 2009, 3, 714-720.

34. Mauter, M. S.; Elimelech, M.; Osuji, C. O. Nanocomposites of Vertically Aligned Single-Walled Carbon Nanotubes by Magnetic Alignment and Polymerization of a Lyotropic Precursor. ACS Nano 2010, 4, 6651-6658.

35. Volkov, A. N.; Zhigilei, L. V. Structural Stability of Carbon Nanotube Films: The Role of Bending Buckling. ACS Nano 2010, 4, 6187-6195.

36. Hahm, M. G.; Wang, H.; Jung, H.; Hong, S.; Lee, S.-G.; Kim, S.-R., Upmanyu, M.; Jung, Y. J. Bundling Dynamics Regulates the Active Mechanics and Transport in Carbon Nanotube Networks. 2011 arXiv:1108.2062v1. arXiv.org e-Print archive. http://arxiv.org/abs/1108.2062 (accessed August 9, 2011).

37. Lipomi, D. J.; Vosgueritchian, M.; Tee, B. C.-K.; Hellstrom, S. L.; Lee, J. A.; Fox, C. H.; Bao, Z. Skin-Like Pressure and Strain Sensors Based on Transparent Elastic Films of Carbon Nanotubes. Nature Nanotechnol. 2011, 6, 788792.

38. Fuhrer, M. S.; Nygard, J.; Shih, L.; Forero, M.; Yoon, Y.-G.; Mazzoni, M. S.; Choi, H. J.; Ihm, J.; Louie, S. G.; Zettl, A.; et al. Crossed Nanotube Junctions. Science 2000, 288, 494-497.

39. Fagan, J. A.; Simpson, J. R.; Landi, B. J.; Richter, L. J.; Mandelbaum, I.; Bajpai, V.; Ho, D. L.; Raffaelle, R.; Hight Walker, A. R.; Bauer, B. J.; et al. Dielectric Response of Aligned Semiconducting Single-Wall Nanotubes. Phys. Rev. Lett. 2007, 98, 147402. 